\title{
Kommunale Akteure verstehen: Verteilte Wissensbestände in Kommunen beachten
}

\author{
Thorsten Erl, Monika Gonser, Jochen Eckart, Elke Häußler \& Jan Riel
}

\section{Herausforderungen bei der Analyse kommunaler Akteure in Reallaboren}

Kommunen spielen in Reallaboren und in der transdisziplinären und transformativen Forschung allgemein oft eine zentrale Rolle. Aufbau und Organisation einer Kommune sind durchaus komplex und können von jeweils ortsspezifischen Besonderheiten geprägt sein. Insofern ist ein besseres Verständnis von Kommunen und insbesondere den einzelnen Akteuren innerhalb einer Kommune (Fachverwaltung, Verwaltungsspitze, politische Entscheidungsträger) von maßgeblicher Bedeutung für das Gelingen der Zusammenarbeit in solchen Projekten.

Für das bessere Verständnis der kommunalen Akteure in Reallaboren und in anderen transdisziplinären und transformativen Forschungsvorhaben bietet sich das Instrument der Akteursanalyse an (Eckart et al. 2018). Die Akteursanalyse hat sich aus Ansätzen der Politikfeldanalyse (Blum und Schubert 2011), des Strategic Management (Freeman 1984) sowie der Partizipationsforschung (Reed et al. 2009; Zimmermann 2006) entwickelt. Auch im Forschungskontext wird die Akteursanalyse bereits angewandt (Butterworth et al. 2011). Sie dient als Instrument, um die Einbindung der Praxisakteure in transdisziplinären und transformativen Forschungsvorhaben im Detail zu planen. Im Rahmen der Akteursanalyse sind die für die Forschungs-, Praxis- und Bildungsziele eines Vorhabens erforderlichen Akteure (Beecroft et al. 2018) zu identifizieren. Die Akteursanalyse befasst sich zudem mit den Erwartungen der Akteure darüber, wie und in welchem Umfang sie sich an einem Vorhaben beteiligen können oder sollen sowie mit den Möglichkeiten und Grenzen der Akteure. Damit eine Akteursanalyse gelingen kann und in der Folge die Zusammenarbeit in einem Projekt fruchtbar verläuft, ist es wichtig, den Besonderheiten der analysierten Akteure Rechnung zu tragen und nicht mit verkürztem Blick an die Analyse heranzugehen. Es ist also, mit anderen Worten, ein Bewusstsein nötig für mögliche ,blinde Flecken', die die Qualität einer Akteursanalyse beeinträchtigen könnten. 
Basierend auf Erfahrungen aus den vom Ministerium für Wissenschaft, Forschung und Kunst Baden-Württemberg (MWK) seit 2015 geförderten 14 Reallaboren der Förderlinien „Reallabore“ und „Reallabore Stadt" (im Folgenden BaWü-Labs) ${ }^{1}$ und bezogen auf den Akteur Kommune wird dargestellt, inwiefern ,blinde Flecken' existieren können, also Wissenslücken auf Seiten der Wissenschaftler(innen) über die Funktionsweise von und die Arbeit mit verschiedenen kommunalen Akteuren. Diese ,blinden Flecken' können in der Akteursanalyse sowie in der eigentlichen Forschungsarbeit zu empfindlichen Komplikationen führen - sei es, weil nicht die richtigen Ansprechpartner gefunden, weil vorgegebene Entscheidungswege nicht beachtet oder weil ohnehin vorbelastete Beziehungen zwischen Akteuren aus Unkenntnis überstrapaziert werden.

Diesen ,blinden Flecken' sind in diesem Buch drei Kurzbeiträge gewidmet: Ein Beitrag stellt die komplexen Entscheidungswege innerhalb kommunaler Strukturen dar, deren Kenntnis für das Gelingen der Zusammenarbeit von besonderer Bedeutung ist (Gonser et al. 2019 in diesem Buch). Ein Beitrag widmet sich der Vorgeschichte kommunaler Akteure und zeigt, warum es wichtig ist, sich mit dieser auseinanderzusetzen (Eckart et al. 2019 in diesem Buch). Der vorliegende Beitrag beschreibt die Verteilung von Wissensbeständen innerhalb von Koтmunen und inwiefern Kommunen nicht nur als ein (homogener) Ansprechpartner (miss)zu verstehen sind.

\section{Strukturierung von Wissen in Fachämtern}

Wissensbezogene, inhaltliche Arbeit in Kommunen wird in Deutschland auf der operativen Ebene von den sogenannten „Fachämtern“ geleistet (analoge Einrichtungen gibt es auch in anderen Ländern, hier wird der in Deutschland gebräuchliche Begriff verwendet). Je größer die Kommune, desto größer und spezialisierter sind in der Regel die Fachämter. Die Fachämter haben innerhalb ihres thematischen Zuständigkeitsbereichs eine beachtliche Entscheidungskompetenz und können Prozesse maßgeblich unterstützen oder blockieren. Folgende Strukturprinzipien werden dabei deutlich, die analog auch für andere Länder als Deutschland gelten dürften:

Kommunale Entscheidungen werden auf der Grundlage von durch die Fachämter aufbereitetem Wissen getroffen: Der Gemeinde- oder Stadtrat, aber auch (Ober-) Bürgermeister(innen) treffen weitreichende Entscheidungen vor allem auf Basis von durch die Fachämter bereitgestelltem Wissen. Informations- bzw. Beschlussvorlagen des Gemeinde- oder Stadtrats werden zum Beispiel durch Fachämter vorbereitet, wobei meist einem Fachamt die Federführung bei der Bearbeitung

1 Für Informationen $\mathrm{zu}$ den beiden Förderlinien siehe http://www.reallabore-bw.de (zugegriffen am 20.05.2019). 
zugewiesen wird. Handelt es sich um eine Maßnahme bzw. um ein Thema beispielsweise eines städtischen Freiraums, wird das Landschaftsamt federführend sein, handelt es sich um eine Mobilitätsfrage, wird die Verkehrsplanung maßgeblich sein etc. Die Wissensauswahl und -bewertung durch die Fachämter ist deswegen von großer politischer Bedeutung. Welches Wissen Fachämtern zur Verfügung steht, auf das sie dabei greifen (können), ist daher auch bedeutsam, wenn es darum geht, Informations- oder Beschlussvorlagen mit Blick auf die Zusammenarbeit mit einem transdisziplinären und transformativen Forschungsprojekt wie einem Reallabor vorzubereiten.

Vernetzungen zwischen den in verschiedenen Fachämtern vorliegenden Wissensbeständen werden durch Methoden des Verwaltungsmanagements hergestellt: Kommunale Verwaltungen sind hierarchisch nach Zuständigkeiten organisiert. Einen Einblick in die Zuständigkeitsverteilung z. B. für Deutschland bietet die allgemeine, bundesweit geltende Struktur, die durch die Kommunale Gemeinschaftsstelle für Verwaltungsmanagement (KGSt) nach dem 2. Weltkrieg aufgestellt wurde. Ein System von Ordnungskennziffern unterscheidet hier auf erster Ebene die übergeordneten Ressorts: Hauptverwaltung (1), Finanzverwaltung (2), Rechts-, Sicherheits- und Ordnungsverwaltung (3), Schul- und Kulturverwaltung (4), Sozial-, Jugend- und Gesundheitsverwaltung (5), Bauverwaltung (6), Verwaltung für öffentliche Einrichtungen (7) und Verwaltung für Wirtschaft und Verkehr (8). Auf einer zweiten Ebene werden diese Ressorts weiter differenziert, wie z. B. in Schulverwaltung (40) und Kulturamt (41) oder in Stadtplanung (61), Bauordnungsamt (63) und Grünflächenamt (67). Dieses System ist für die Kommunen in Deutschland nicht zwingend anzuwenden, jedoch häufig vorzufinden. Im Ergebnis verfügen die Mitarbeitenden in der kommunalen Verwaltung nur in ihrem jeweils thematisch eingeschränkten (Zuständigkeits-)Bereich über vertieftes Wissen. Den Akteuren der kommunalen Verwaltung ist diese ,Wissensordnung' wohlbekannt, für Wissenschaftler(innen) hingegen ist sie zwar wichtig, wenn sie mit Kommunen zusammenarbeiten, aber zumeist nicht oder nur marginal bekannt.

Im Reallabor GO Karlsruhe - Partizipative Forschung für den Fußgängerverkehr (,,BaWü-Lab GO Karlsruhe“) war das für die Arbeit im Projekt erforderliche kommunale Fachwissen über Stadtplanungsamt (Verkehrsplanung, inhaltliche Arbeit an der Bürgerbeteiligung), Tiefbauamt, Ordnungsamt (in der Rolle als Straßenverkehrsbehörde), eine ämterübergreifende Baustellenkoordination, Stadtmarketing sowie das Amt für Stadtentwicklung (als organisierende Stelle für Bürgerbeteiligung) breit verteilt. Aufgrund der früheren Mitarbeit eines Mitglieds des Forscher(innen)teams in der Stadtverwaltung waren jedoch Kenntnisse über die Organisation der Ämter wie auch über persönliche Ansprechpartner vorhanden, so dass der Zugriff auf das relevante Fachwissen auch ohne vorhergehende umfangreiche Akteursanalyse einfach möglich war. Wäre das nicht der Fall gewesen, wäre eine umfassende Analyse nötig gewesen, um herauszufinden, welche Fachämter über das jeweils relevante Wissen verfügen. 
Bei kleineren Kommunen besteht dagegen möglicherweise aufgrund der Bündelung verschiedener Ressorts in einer Person (Bürgermeister(in), Amtsleitung) bereits bei dieser Person umfangreiches Querschnittswissen, was es entsprechend einfacher macht, an dieses Wissen heranzukommen.

Das Reallabor STADT-RAUM-BILDUNG - Reallabor für die nachhaltige Planung von Bildungslandschaften und die Integration von Aus- und Umbauten von Schulgebäuden (,,BaWü-Lab SRB“) (s. auch Steckbrief im Anhang zu diesem Buch) arbeitete mit unterschiedlich großen Kommunen. Während in Heidelberg eine kontinuierliche Kommunikation mit drei Amtsleitungen (Stadtplanungsamt, Gebäudemanagement, Amt für Schule und Bildung) aufgeteilt auf zwei Dezernate (Dezernat für Bauen und Verkehr, Dezernat für Familie, Soziales und Kultur) wichtig war, kommunizierten die Wissenschaftler(innen) in der Landeshauptstadt Stuttgart mit dem Schulverwaltungsamt, das eine eigene Planungsabteilung für den Schulbau unterhält. In Müllheim wiederum wurde hauptsächlich mit der Leitung des Baudezernats zusammengearbeitet.

Die Vernetzung zwischen verschiedenen (Zuständigkeits-)Bereichen und Wissensbeständen wird im Verwaltungsalltag meist nur von Personen in Führungspositionen wahrgenommen. Über die Vernetzung durch Führungspositionen hinaus gibt es in kommunalen Verwaltungen noch andere Werkzeuge zur Wissensverknüpfung, von denen einige hier beispielhaft genannt seien: Der Austausch zwischen den einzelnen Ressorts bzw. Fachämtern funktioniert gemeinhin über den sogenannten „Ämterumlauf“. Mittels einer Akte zum Sachverhalt werden die entsprechenden Ämter in Kenntnis gesetzt und um Stellungnahme gebeten. Diese Stellungnahmen werden nach einer gewissen Bearbeitungszeit wieder an das federführende Amt zurückgeschickt. Daraufhin muss dieses Amt alle Hinweise, das heißt, das Wissen der anderen Ämter, aufarbeiten und in die Vorlage einpflegen. Zudem versuchen einige Verwaltungen, die hierarchischen Strukturen über sogenannte ,agile“ Arbeitsgruppen fachämterübergreifend themen- bzw. projektbezogen aufzubrechen und neu zu strukturieren. Damit werden in der Verwaltung erste Schritte unternommen, um gerade auch für z. B. Reallabore relevantes Querschnittswissen zu erzeugen. Für ein transdisziplinäres und transformatives Projekt bedeutet das, dass nur an wenigen Stellen in einer kommunalen Verwaltung integriertes Querschnittswissen vorliegen dürfte und dass es nicht nur herauszufinden gilt, wo sich welches Wissen bündelt, sondern auch, wie innerhalb der Kommune Wissen ausgetauscht und gebündelt wird.

\section{Herausforderungen im Umgang mit kommunalem Wissen}

Das Arbeiten mit kommunalen Wissensbeständen gestaltet sich aufgrund der beschriebenen komplexen und hierarchischen kommunalen Verwaltungsstrukturen in Teilen als langwierig bzw. ,schwerfällig'. Für ,Externe', wie etwa Wissenschaftler(innen) in einem Reallabor, kann es sich überaus schwierig gestalten, zu 
erkennen, welche Wissensbestände in den Fachämtern vorhanden sind, wie sich diese gliedern und wo und wie Wissen gebündelt wird. Weiter wird dies durch die fast wie ,Geheimcodes' erscheinende Verwaltungsgliederung erschwert, die für außenstehende Wissenschaftler(innen) auf den ersten Blick nur schwer erfassbar ist.

Probleme entstehen insbesondere dann, wenn in einem transdisziplinären und transformativen Projekt wie einem Reallabor davon ausgegangen wird, dass alle Akteure in der kommunalen Verwaltung über das gleiche Wissen verfügen bzw. dass alles für das Reallabor relevante Wissen bei einem Akteur innerhalb der kommunalen Verwaltung zu finden ist. Das ,unterkomplexe' Verständnis der Verteilung von Wissensbeständen innerhalb einer Kommune wird so zu einem ,blinden Fleck'.

\section{Berücksichtigung der verteilten Wissensbestände in der Akteursanalyse}

Im Rahmen der Akteursanalyse ist deshalb zu identifizieren, welche Akteure in der kommunalen Verwaltung über welche Teilaspekte des für das Projekt relevanten Wissens verfügen. Zudem ist zu klären, ob es Stellen in der Verwaltung gibt, die das für das Projekt relevante Querschnittswissen zusammenfassen. Dabei ist davon auszugehen, dass die Wissensbestände auf eine Vielzahl von Akteuren verteilt sind und nicht mit einem Ansprechpartner allein erschlossen werden können. Für Deutschland kann die oben erwähnte Zuständigkeitsverteilung gemäß KGSt erste Ansatzpunkte liefern, die relevanten Ansprechpartner innerhalb der kommunalen Verwaltung zu identifizieren. Teilweise werden allerdings in den Kommunen die Zuschnitte der einzelnen Ressorts den örtlichen Besonderheiten angepasst (und auch anders benannt). Diese lokalen Anpassungen wiederum können im Organigramm der Kommune sowie durch die genauere Beschreibung der Zuständigkeitsverteilung innerhalb der Verwaltung in Erfahrung gebracht werden.

Um als transdisziplinäres und transformatives Projekt auf kommunale (Querschnitts-)Wissensbestände zugreifen zu können, ist es notwendig, auch innerhalb der Kommune Ansprechpartner oder Teams zu identifizieren bzw. zu bilden, die den seitens der Wissenschaft gewählten gesamtheitlichen Zugang angemessen spiegeln. Insbesondere bestehende fachämterübergreifende Teams können helfen, innerhalb der Verwaltung Querschnittswissen zu erschließen und in das gemeinsame Forschungsprojekt einzubringen. Falls es keine solchen gibt, lohnt es sich, in Erfahrung zu bringen, ob eine solche Arbeitsgruppe eingerichtet werden könnte. Im besten Fall ist das transdisziplinäre Team ,symmetrisch' organisiert, d. h., dass sich jeweils ein(e) Vertreter(in) aus einem kommunalen Fachamt und ein(e) 
Vertreter(in) aus der Wissenschaft entsprechen, was Themenschwerpunkte der Arbeit und Wissenschaftskultur betrifft. In diesem Fall ist zumindest gewährleistet, dass die gemeinsame Arbeit auf der Grundlage ähnlicher Sprachen und ähnlicher Arbeitskulturen aufbauen kann. Das erhöht theoretisch die Möglichkeit, im Verlauf des Projekts zwischen kommunalen Akteuren und Wissenschaftler(inne)n ,auf Augenhöhe' diskutieren und forschen zu können. Kritisch ist vor diesem Hintergrund ein ,asymmetrisches" Verhältnis, d. h., wenn Einzelpersonen aus sehr unterschiedlichen Wissenschaftskulturen (z. B. Geisteswissenschaften und Ingenieurwissenschaften) aufeinandertreffen. In solchen Fällen sollte versucht werden, breiter aufgestellte Teams einzusetzen, so dass das ausgetauschte Fachwissen auch von allen Beteiligten richtig verstanden und interpretiert wird. In einer umfassenden Akteursanalyse sollte deshalb auch beleuchtet werden, welchen fachlichen Hintergrund die zuständigen Personen in der kommunalen Verwaltung haben, d. h., auch diesem möglichen ,blinden Fleck' sollte Aufmerksamkeit geschenkt werden.

\section{Literatur}

Beecroft, R., Trenks, H., Rhodius, R., Benighaus, C., \& Parodi, O. (2018). Reallabore als Rahmen transformativer und transdisziplinärer Forschung: Ziele und Designprinzipien. In R. Defila \& A. Di Giulio (Hrsg.), Transdisziplinär und transformativ forschen. Eine Methodensammlung. (S. 75-100). Wiesbaden: Springer VS. doi: 10.1007/978-3-65821530-9_4.

Blum, S., \& Schubert, K. (2011). Politikfeldanalyse. Wiesbaden: Springer VS.

Butterworth, J., McIntyre, P. \& da Silva Wells, C. (Hrsg.). (2011). Switch in the City: Putting Urban Water Management to the Test. Den Haag: IRC International Water and Sanitation Centre.

Eckart, J., Ley, A., Häußler, E., \& Erl, Th. (2018). Leitfragen für die Gestaltung von Partizipationsprozessen in Reallaboren. In R. Defila \& A. Di Giulio (Hrsg.), Transdisziplinär und transformativ forschen. Eine Methodensammlung (S. 105-135). Wiesbaden: Springer VS.

Freeman, R. E. (1984). Strategic Management: A Stakeholder Approach. Boston: Pitman.

Reed, M. S., Graves, A., Dandy, N., Posthumus, H., Hubacek, K., \& Morris, J. (2009). Who's in and why? A typology of stakeholder analysis methods for natural resource management. Journal of Environmental Management, 90, (S. 1933-1949).

Zimmermann, A. (2006). Instrumente zur Akteursanalyse - 10 Bausteine für die partizipative Gestaltung von Kooperationssystemen. Eschborn: Deutsche Gesellschaft für Technische Zusammenarbeit (GTZ). 


\section{Die zwei weiteren Beiträge in diesem Buch zu, blinden Flecken ‘}

Eckart, J., Häußler, E., Erl, Th., Gonser, M., \& Riel, J. (2019). Kommunale Akteure verstehen: Vorgeschichte der Arbeit in Kommunen beachten. In R. Defila \& A. Di Giulio (Hrsg.), Transdisziplinär und transformativ forschen, Band 2. Eine Methodensammlung (S. 33-38). Wiesbaden: Springer VS.

Gonser, M., Riel, J., Eckart, J., Erl, Th., \& Häußler, E. (2019). Kommunale Akteure verstehen: Komplexe Entscheidungswege in Kommunen. In R. Defila \& A. Di Giulio (Hrsg.), Transdisziplinär und transformativ forschen, Band 2. Eine Methodensammlung (S. 85-91). Wiesbaden: Springer VS.

Open Access Dieses Kapitel wird unter der Creative Commons Namensnennung 4.0 International Lizenz (http://creativecommons.org/licenses/by/4.0/deed.de) veröffentlicht, welche die Nutzung, Vervielfältigung, Bearbeitung, Verbreitung und Wiedergabe in jeglichem Medium und Format erlaubt, sofern Sie den/die ursprünglichen Autor(en) und die Quelle ordnungsgemäß nennen, einen Link zur Creative Commons Lizenz beifügen und angeben, ob Änderungen vorgenommen wurden.

Die in diesem Kapitel enthaltenen Bilder und sonstiges Drittmaterial unterliegen ebenfalls der genannten Creative Commons Lizenz, sofern sich aus der Abbildungslegende nichts anderes ergibt. Sofern das betreffende Material nicht unter der genannten Creative Commons Lizenz steht und die betreffende Handlung nicht nach gesetzlichen Vorschriften erlaubt ist, ist für die oben aufgeführten Weiterverwendungen des Materials die Einwilligung des jeweiligen Rechteinhabers einzuholen.

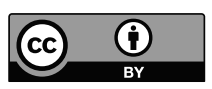

